\title{
Tire Tread Pattern Recognition and Feature Analyzing Based on Hu Moment
}

\author{
Fan Zhang ${ }^{1, a}$, Puwang $\mathrm{Li}^{1, b,{ }^{*}}$, Yongzhen $\mathrm{Li}^{1, \mathrm{c}}$, Yijun Liu${ }^{1, \mathrm{~d}}$, Ziming Yang ${ }^{1, \mathrm{e}}$ \\ ${ }^{1}$ Agricultural Product Processing Research Institute at Chinese Academy of Tropical Agricultural \\ Sciences, Chinese Agricultural Ministry Key Laboratory of Tropical Crop Products Processing, \\ Zhanjiang 524001, P.R. China \\ afanzhang1024@163.com, bpuwangli@163.com, clyz_228@163.com, dliuyijun-1@163.com, \\ yangziming2004@ \\ ${ }^{*}$ Corresponding author
}

Keywords: Tire Tread Pattern, Recognition, Feature Analyzing, Hu moment.

\begin{abstract}
This paper aims to recognize the tire tread pattern from DXF (Drawing Interchange Format) file and to analyze its basis feature. A DXF file analyzing algorithm is used to obtain the data according to the formulate of DXF; a feature analyzing approach based on $\mathrm{Hu}$ moment is utilized to compute the seven characteristic moments and to confirm the invariance of pattern image under image rotation, scaling, and translation; a feature analyzing system is developed by Java to show the basic feature. The experimental results show that the proposed system has a good achievement and can get a series of ideal parameters for the further research.
\end{abstract}

\section{Introduction}

Tire tread pattern, as one of the most important part of a tire, has three major functions such as enhancing the grip, reducing noise and increasing drainage. As these functions constraint each other, it's difficult to design a set of perfect pattern to satisfy all functions. In the past, designing pattern closely depended on manual labor to input basic information, which may bring lots of errors. With the development of computation techniques, computer aided system are playing more important role in the process of designing tread pattern. Many researchers focused on studying tire tread pattern recognition and matching by designing algorithms. Yu Qing proposed tire tread pattern retrieval algorithm to identify the scene tire information by comparing the pattern images with database patterns [1]. Dong Yude studied boundary features extraction and proposed an algorithm to reconstruct tire tread patterns [2]. These researches mainly study tire tread pattern with images in different application background. However, it's also important to recognize the pattern in the design process.

AutoCAD is the most popular software to design tread pattern, and whose users usually output plane graph with DWG and DXF files in two formats. It is a typical engineering problem that analyzing the data of DXF files to interact with other programs, which has appealed many researchers. Huibin Yang proposed a graphic information extraction method to transform the graphic information identified from DXF file into bottom motion controller's codes which can drive the engraving machine [3]. Chen Shumin and Fan Zhang analyzed DXF files and extracted the coordinate data, then generated NC codes for the cutting path of layout results $[4,5]$. In short, studying the DXF file is advantageous to the second development of drawing software.

$\mathrm{Hu}$ moment, firstly proposed by $\mathrm{Hu}[6]$, has been widely utilized to image pattern recognition. Proved to be invariant to scaling, translation, and rotation, Hu moment plays good robustness and reliability for recognizing image. Many researchers used $\mathrm{Hu}$ invariant moment to solve practical problem. $\mathrm{Xu}$ xiaosu used $\mathrm{Hu}$ moment to matching two-dimensional terrain feature in an underwater assisted navigation system [7]. Zou Xiuguo used Hu moment based DSP (Digital Signal Processing) hardware platform for face recognition [8]. 
This paper focuses on developing a pattern feature analyzing system that caters for designing tire tread pattern. Firstly, a DXF analyzing algorithm based on Java is discussed to obtain the basic pattern information (Section 2); next, the invariance of tread pattern is analyzed based on Hu moment, and then a pattern feature analyzing system, developed based on the above method, is described and introduced about the simple application in the design process(Section 3); at last, section 4 makes a discussion and conclusion of this work.

\section{Recognition algorithm for pattern DXF file}

The complete structure of DXF includes seven segments called SECTION which contains HEADER, CLASSES, TABLES, BLOCKS, ENTITIES, OBJECTS, and EOF. While the ENTITIES section includes any block references including LINE, LWPOLYLINE, CIRCLE, ARC, ELLIPSE and SPLINE. These ENTITIES objects store information with the format of group code and its corresponding group value. Table 1 shows the detail information of all the ENTITIES objects.

Table 1. The description of group code

\begin{tabular}{|c|c|c|c|c|c|}
\hline ENTITIES & Group code & Description & ENTITIES & Group code & Description \\
\hline \multirow{2}{*}{ CIRCLE } & $10,20,30$ & Center point $(\mathrm{x}, \mathrm{y}, \mathrm{z})$ & \multirow{2}{*}{ LINE } & $10,20,30$ & Start-point $(\mathrm{x}, \mathrm{y}, \mathrm{z})$ \\
\hline & 40 & Radius & & $11,21,31$ & End-point $(\mathrm{x}, \mathrm{y}, \mathrm{z})$ \\
\hline \multirow{5}{*}{ LWPOLYLINE } & 90 & The number of vertex & \multirow{5}{*}{$\begin{array}{l}\text { POLYLIN } \\
\text { E }\end{array}$} & 70 & 1 for close, 0 for open \\
\hline & 70 & 1 for close, 0 for open & & 0 & Start-point tag \\
\hline & 10,20 & $\operatorname{Vertex}(\mathrm{x}, \mathrm{y})$ & & $10,20,30$ & Point $(x, y, z)$ \\
\hline & 42 & Convexity of vertex & & 42 & $\operatorname{Vertex}(\mathrm{x}, \mathrm{y})$ \\
\hline & 43 & linewidth & & 0 & End-point tag \\
\hline \multirow{4}{*}{ ARC } & $10,20,30$ & Center point $(\mathrm{x}, \mathrm{y}, \mathrm{z})$ & \multirow{4}{*}{ ELLIPSE } & $10,20,30$ & Center point $(\mathrm{x}, \mathrm{y}, \mathrm{z})$ \\
\hline & 40 & Radius & & $11,21,31$ & Macroaxis endpoint \\
\hline & 50 & Start angle & & 40 & Macroaxis or minor axis \\
\hline & 51 & End angle & & 41,42 & Start angle, end angle \\
\hline \multirow{2}{*}{ SPLINE } & $71,72,73$ & $\begin{array}{l}\text { times, nodes and control } \\
\text { points of NURBS }\end{array}$ & \multirow{2}{*}{ SPLINE } & $10,20,30$ & Control point(x, y, z) \\
\hline & 40,41 & $\begin{array}{c}\text { Node value, weight factor } \\
\text { value }\end{array}$ & & $11,21,31$ & Fitting point $(x, y, z)$ \\
\hline
\end{tabular}

According to the above characteristics, a recognition algorithm can be proposed to acquire the block information of pattern DXF file. Under the basic theory of string matching, this algorithm can get a set of blocks data that involve circles, lines, splines, and so on. From these data, a gray image of pattern DXF file can be generated. Fig. 1 shows an example of tire tread pattern and its gray image.
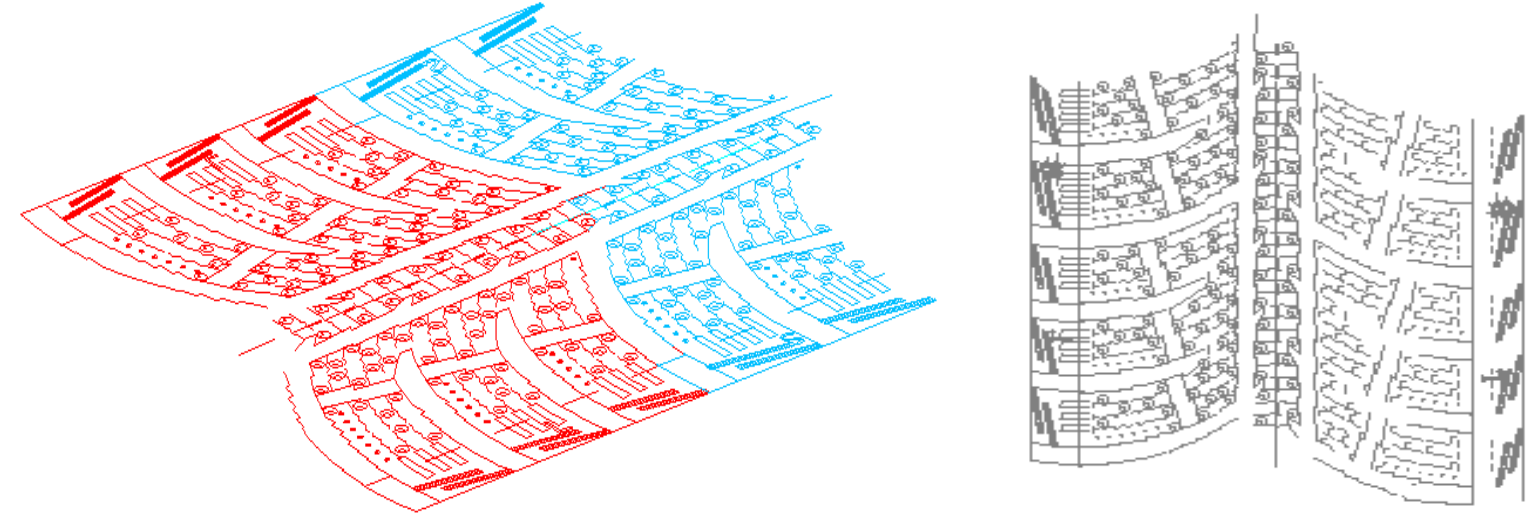

Fig. 1 The DXF file of tire tread Pattern in CAD (left) and the generated gray image after processing (right)

\section{Feature analyzing approach based on Hu moment}

The invariant moments is often used to describe the parameter of image features. Based on the area moment invariant theory, 7 invariant moments related to translation, rotation, scale and scale (called 
Hu moments) are constructed, which can describe the shape of the spatial region. These 7 invariant moments have become important regional feature sets, and have been widely used in image recognition and pattern analysis.

For the two-dimensional continuous distribution $f(x, y)$, the $(p+q)$ th moment of the area $\zeta$ can be defined as follows:

$$
m_{p q}=\iint_{\varsigma} x^{p} y^{q} f(x, y) d x d y \quad(p, q=0,1,2, \ldots)
$$

Then the central moment can be calculated as follows:

$$
u_{p q}=\iint_{\varsigma}(x-\bar{x})^{p}(y-\bar{y})^{q} f(x, y) d x d y \quad(p, q=0,1,2, \ldots)
$$

Where $\bar{x}$ and $\bar{y}$ are the centroid coordinates of the image $f(x, y)$, which are defined as follows:

$$
\bar{x}=m_{10} / m_{00} \quad \bar{y}=m_{01} / m_{00}
$$

While for the discrete distribution $f(x, y)$, then the formula (1) and formula (2) can be deformed as follows:

$$
m_{p q}=\sum_{x}^{H} \sum_{y}^{W} x^{p} y^{q} f(x, y), \quad u_{p q}=\sum_{x}^{H} \sum_{y}^{W}(x-\bar{x})^{p}(y-\bar{y})^{q} f(x, y)
$$

Where $H$ is the height of image, and $W$ is the width of image. Then the normalized central moments can be described as follows:

$$
\eta_{p q}=u_{p q} / u_{00}^{\lambda}, \lambda=(p+q+2) / 2, p+q=2,3, \ldots
$$

From the above formula, $\mathrm{Hu}$ constructed seven moment invariants by using second and third normalized central moments as follows:

$$
\begin{aligned}
& M_{1}=\eta_{20}+\eta_{02} \\
& M_{2}=\left(\eta_{20}-\eta_{02}\right)^{2}+4 \eta_{11} \\
& M_{3}=\left(\eta_{30}-3 \eta_{12}\right)^{2}+\left(3 \eta_{21}-u_{03}\right)^{2} \\
& M_{4}=\left(\eta_{30}+\eta_{12}\right)^{2}+\left(\eta_{21}+u_{03}\right)^{2} \\
& M_{5}=\left(\eta_{30}-3 \eta_{12}\right)\left(\eta_{30}+\eta_{12}\right)\left[\left(\eta_{30}+\eta_{12}\right)^{2}-3\left(\eta_{21}+\eta_{03}\right)^{2}\right]+\left(3 \eta_{21}-\eta_{03}\right)\left(\eta_{21}+\eta_{03}\right)\left[3\left(\eta_{30}+\eta_{12}\right)^{2}-\left(\eta_{21}+\eta_{03}\right)^{2}\right] \\
& M_{6}=\left(\eta_{20}-\eta_{02}\right)\left[\left(\eta_{30}+\eta_{12}\right)^{2}-\left(\eta_{21}+\eta_{03}\right)^{2}\right]+4 \eta_{11}\left(\eta_{30}+\eta_{12}\right)\left(\eta_{21}+\eta_{03}\right) \\
& M_{7}=\left(3 \eta_{21}-\eta_{03}\right)\left(\eta_{30}+\eta_{12}\right)\left[\left(\eta_{30}+\eta_{12}\right)^{2}-3\left(\eta_{21}+\eta_{03}\right)^{2}\right]-\left(\eta_{30}-3 \eta_{12}\right)\left(\eta_{21}+\eta_{03}\right)\left[3\left(\eta_{30}+\eta_{12}\right)^{2}-\left(\eta_{21}+\eta_{03}\right)^{2}\right]
\end{aligned}
$$

These seven moment invariants compose a set of characteristic vector, which have the feature of invariance under image rotation, scaling, and translation. According to these feature of Hu moment, we input the DXF file of tire tread Pattern as Fig.1, and calculate these moments. Rotation, scaling and translation are also be tested by the above method. Table 2 shows the results of experiment.

Table 2. The result of experiment for rotation, scaling and translation

\begin{tabular}{ccccc}
\hline Huments & Original image & Rotated image & Scaled image & Translated image \\
\hline $\mathrm{M}_{1}$ & 2.5462 & 2.4982 & 2.4484 & 2.4863 \\
$\mathrm{M}_{2}$ & 4.0032 & 4.0041 & 4.0038 & 4.0296 \\
$\mathrm{M}_{3}$ & 0.0309 & 0.0193 & 0.0227 & 0.0305 \\
$\mathrm{M}_{4}$ & 0.0271 & 0.0163 & 0.0463 & 0.0362 \\
$\mathrm{M}_{5}$ & 0.0001 & 0.0002 & -0.0012 & -0.0009 \\
$\mathrm{M}_{6}$ & -0.0396 & -0.0330 & -0.0305 & -0.0218 \\
$\mathrm{M}_{7}$ & -0.0007 & 0.0001 & 0.0008 & -0.0007 \\
\hline
\end{tabular}

Based on the above method, a pattern feature analyzing system is developed by Java to describe the simple application in the design process. The system is shown as Fig. 2 shows. 


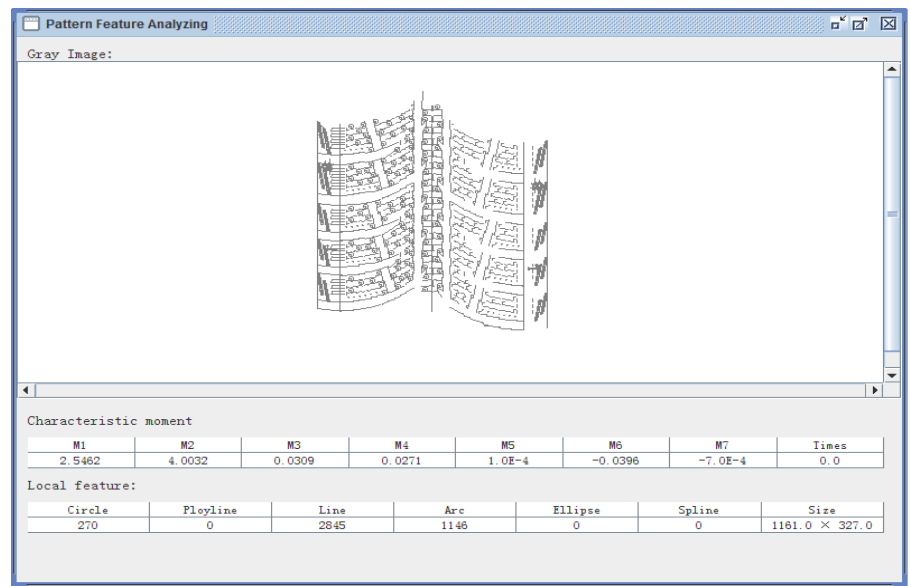

Fig. 2 The pattern feature analyzing system

\section{Summary}

This paper presents a set of method about the recognition and feature analyzing of tire trend pattern. A DXF analyzing algorithm is used to recognize the information of tire trend pattern, and a feature analyzing approach based on Hu moments is used to dissect the feature of pattern. At last, a system is developed based on above method, and an experimental results show that the system has a good achievement.

Future work will focused on the matching and reconstruction for tire tread patterns.

\section{Acknowledgement}

This research was financially supported by the Natural Science Foundation of Hainan Province, China (Grant No. 317248), and Central Public-interest Scientific Institution Basal Research Fund for Chinese Academy of Tropical Agricultural Sciences (Grant No. 1630122017010),

\section{References}

[1] Q. Yu, Research on scene tire tread pattern retrieval algorithm, Dalina Maritime University, 2015.

[2] Y. Dong, R. Zhang, Z. Song, Y. Fang, S. Bai, F. Zhang, Research on Boundary Features Extraction and Reconstruction for Tire Tread Patterns, Journal of Mechanical Engineering, 2017.

[3] H. Yang. DXF File Identification with C\# for CNC Engraving Machine System. Intelligent Control and Automation, vol.6, pp. 20-28, 2015.

[4] S. Chen, Q. Liu, DXF applications to layout cutting, Computer Applications and Software, vol. 29, pp.143-187, 2012.

[5] F. Zhang, J. Gong, Y. Liu, A geometry -based path optimization approach for motion control of the NC grinding machine, Civil, Architecture and Environmental Engineering, pp.621-624,2017.

[6] H. Ming-kuei, Visual pattern recognition by moment invariants, Information Theory, vol. 8, pp.179-187,1962.

[7] X. Xu, Z. Yue, T. Zhang, J. Tang, Method on underwater assisted navigation system using two-dimensional terrain feature matching based on $\mathrm{Hu}$ moments, Journal of Chinese Inertial Technology, vol. 23, pp. 363-368, 2015.

[8] X. Zou, L. Li, J. Lu, Research of face recognition Hu moment based on DSP, Computer Technology and Its Applications, vol. 38, pp.150-156, 2012. 